\title{
Assessment of Macro-, Micro-, Trace, and Ultratrace Element Concentration in Green-Legged Partridge Hens' Eggs from a Free-Range System
}

\author{
Tadeusz Trziszka ${ }^{1}$, Zbigniew Dobrzański ${ }^{2}$, Katarzyna Chojnacka ${ }^{3}$, Anna Bubel ${ }^{2}$, Halina Beń ${ }^{1}$, \\ Mariusz Korczyński ${ }^{4}$ (D), Damian Konkol ${ }^{4, *}$ and Wojciech Tronina ${ }^{5}$
}

Citation: Trziszka, T.; Dobrzański, Z.; Chojnacka, K.; Bubel, A.; Beń, H.; Korczyński, M.; Konkol, D.; Tronina, W. Assessment of Macro-, Micro-, Trace, and Ultratrace Element Concentration in Green-Legged Partridge Hens' Eggs from a Free-Range System. Agriculture 2021, 11,473. https://doi.org/10.3390/ agriculture11060473

Academic Editor:

Agnieszka Ludwiczak

Received: 28 April 2021

Accepted: 20 May 2021

Published: 21 May 2021

Publisher's Note: MDPI stays neutral with regard to jurisdictional claims in published maps and institutional affiliations.

Copyright: (c) 2021 by the authors. Licensee MDPI, Basel, Switzerland. This article is an open access article distributed under the terms and conditions of the Creative Commons Attribution (CC BY) license (https:// creativecommons.org/licenses/by/ $4.0 /)$
1 Department of Functional Food Products Development, Wroclaw University of Environmental and Life Sciences, Chełmońskiego 37, 51-630 Wroclaw, Poland; tadeusz.trziszka@upwr.edu.pl (T.T.); halina.ben@upwr.edu.pl (H.B.)

2 Department of Environment Hygiene and Animal Welfare, Wroclaw University of Environmental and Life Sciences, Chełmońskiego 38C, 51-630 Wroclaw, Poland; zbigniew.dobrzanski@upwr.edu.pl (Z.D.); anna.bubel@upwr.edu.pl (A.B.)

3 Department of Advanced Material Technologies, Wroclaw University of Science and Technology, Smoluchowskiego 25, 50-372 Wroclaw, Poland; katarzyna.chojnacka@pwr.edu.pl

4 Department of Animal Nutrition and Feed Management, Wroclaw University of Environmental and Life Sciences, Chełmońskiego 38C, 51-630 Wroclaw, Poland; mariusz.korczynski@upwr.edu.pl

5 Breeding and Innovative Company Tronina, Raków 34 A, 55-093 Kiełczów, Poland; tronina@tronina.pl

* Correspondence: damian.konkol@upwr.edu.pl; Tel.: +48-663-052-445

Abstract: The goal of this research was to determine the concentration of macro-, micro-, trace, and ultratrace elements in egg albumen and yolk of Green-Legged Partridge (GLP) hens (Polish native breed) maintained in a free-range system. The hens received two feed mixtures (FM), which differed in mineral content (Gr-1 and Gr-2). Analyses of 57 chemical elements were performed using inductively coupled plasma spectroscopy by ICP-OES and ICP-MS techniques. FM from Gr-2 were characterized by a significantly ( $p<0.05$ or 0.01 ) higher concentration of macro- and microelements $(\mathrm{Al}, \mathrm{Ba}, \mathrm{Ca}, \mathrm{K}, \mathrm{P}, \mathrm{S}, \mathrm{Si})$ than that from Gr-1. Among the trace and ultratrace elements there were significantly higher levels of $\mathrm{Co}, \mathrm{Cr}, \mathrm{Li}, \mathrm{Nb}, \mathrm{Ni}, \mathrm{Pb}, \mathrm{Ru}, \mathrm{Sn}$, and $\mathrm{Zr}$, and significantly lower levels of Cs, Pd, and Te in Gr-2 compared with Gr-1. Egg albumen from hens from the Gr-2 group contained a significantly higher Ba concentration. In the case of trace and ultratrace elements, significantly greater $\mathrm{Au}, \mathrm{Co}, \mathrm{Cr}, \mathrm{Mo}, \mathrm{Nb}, \mathrm{Ru}$, and Se, and less As were recorded in the albumen from $\mathrm{Gr}-2$. No significant differences were observed in the concentrations of macro- and microelements in the egg yolk. Among the examined trace and ultratrace elements, substantial differences were observed in the level of $\mathrm{Co}, \mathrm{Cr}, \mathrm{Ni}$, Se, Sn, and Y (more in $\mathrm{Gr}-2$ ), and $\mathrm{Au}, \mathrm{Hf}, \mathrm{Hg}, \mathrm{Nb}, \mathrm{Nd}$, and $\mathrm{Rb}$ (less in $\mathrm{Gr}-2$ ). Only in the case of $\mathrm{Co}$ and $\mathrm{Cr}$ concentrations in the feed (Gr-2) was a significant increase found in the albumen and yolk. Gr-2 also showed a significantly greater concentration of $\mathrm{Ba}, \mathrm{Nb}$, and $\mathrm{Ru}$ in the albumen, and $\mathrm{Ni}$ and $\mathrm{Sn}$ in the yolk.

Keywords: Green-Legged Partridge; free-range system; egg albumen; egg yolk; chemical composition

\section{Introduction}

Eggs are an important source of many nutrients necessary for the proper development and functioning of the human organism. They are a good source of nutraceuticals used in the prevention of civilization diseases [1-3]. The chemical composition of eggs is complex and may be influenced by various factors (breed, age of birds, housing system, and feed composition) [4-6]. The mineral composition of eggs is important in terms of food safety, due to the fact that all elements, including essential, can become toxic or lead to deficiencies if consumed in inappropriate amounts [7-9]. Additionally, feed and environment, and thus eggs, may be contaminated with potentially toxic elements, such as $\mathrm{Ag}$, $\mathrm{As}, \mathrm{Be}, \mathrm{Cd}, \mathrm{Cr}, \mathrm{Hg}$, $\mathrm{Ni}, \mathrm{Pb}, \mathrm{Sb}, \mathrm{V}$, and $\mathrm{Tl}$ [10-12]. 
Reports have described the chemical composition of eggs from different breeds of hens from different housing and feeding systems, but determinations of macro- and trace element contents are limited. For example, determinations of the concentration of 11 microand trace elements $(\mathrm{Ba}, \mathrm{Cu}, \mathrm{Fe}, \mathrm{Mn}, \mathrm{Ni}, \mathrm{Pb}, \mathrm{Rb}, \mathrm{Se}, \mathrm{Sr}, \mathrm{V}$, and $\mathrm{Zn}$ ) in hens eggs obtained from various poultry housing systems in Latvia were reported. Eggs from organic farms had the most variable concentration range and the highest element content, while eggs from other maintenance systems contained lower element concentrations [13]. The content of 12 trace elements ( $\mathrm{As}, \mathrm{Cd}, \mathrm{Co}, \mathrm{Cr}, \mathrm{Cu}, \mathrm{Mo}, \mathrm{Mn}, \mathrm{Ni}, \mathrm{Se}, \mathrm{V}, \mathrm{Ti}$, and $\mathrm{Zn}$ ) in hen eggs (Lohman type) from three maintenance systems was analyzed by Giannenas et al. [14]. The authors concluded that trace element content in yolks were higher than trace element content in albumen. $\mathrm{Cr}$ concentration was higher in a courtyard, rather than organic, diet. The highest concentration of Se in yolks was found in eggs from the organic system, followed by eggs from the conventional system. Zn concentration in yolks was highest in the courtyard system, followed by yolks from the conventional system, which, in turn, was higher than yolks from the organic system. Mn concentration was lowest in yolks from the courtyard system, but $\mathrm{Cr}$ concentration was highest in eggs from the courtyard system. In the case of albumen, there were differences in $\mathrm{Zn}$ and $\mathrm{Cr}$ levels, which were the highest in albumen from the courtyard system. The results show measurable differences among eggs from hens maintained in different production systems.

Other reports determined the level of some trace elements ( $\mathrm{As}, \mathrm{Co}, \mathrm{Cu}, \mathrm{Fe}, \mathrm{Mn}$, and Se) in eggs from three different sources (commercial farm, village, and markets) in Malaysia. The greatest concentration of these metals (except $\mathrm{Co}$ ) was found in eggs from commercial farms, compared with other sources [15]. However, Kiczorowska et al. [5] proved that the mineral composition of Rhode Island Red (RIR) hen eggs varied significantly, depending on the large-scale, courtyard, or organic systems. Free-range eggs showed a substantial increase in the content of macroelements $\mathrm{K}$ and $\mathrm{Na}$ (albumen and yolk) and $\mathrm{Ca}$ and $\mathrm{Mg}$ (yolk), and microelements $\mathrm{Zn}$, Se, and Mn (albumen and yolk) and Fe (yolk). The authors explained this with the possibility of supplementing the diet of hens on the paddock. Additionally, researchers [16] believe that hen type, age, and rearing environment has little effect on the chemical composition of egg content, and the observed differences in mineral content of eggs are unlikely to have significant effect on human nutrition.

It seems that the chemical composition of the feed, both the concentration of macronutrients and trace elements, may have the greatest impact on the mineral composition of eggs. A recent study [6] has determined the level of 38 chemical elements in the egg white and yolk of Lohmann Brown (LB) hens kept in a cage system and fed with commercial feed mixture with additive of humic preparations. The authors showed the limited effect of the mineral composition of feed on the concentration of essential and nonessential elements in eggs. These relationships were significant in the case of $\mathrm{Bi}, \mathrm{Co}, \mathrm{Ni}$, and $\mathrm{S}$ (feed-albumen), and $\mathrm{Bi}, \mathrm{Fe}, \mathrm{K}$, and $\mathrm{Sb}$ (feed-yolk).

Despite many studies on the mineral composition of hen eggs and the influencing factors, there is no information to-date regarding the content of minerals in eggs of local breeds (e.g., Green-Legged Partridge-Polish native breed), which are increasingly kept in ecological, organic, and free-range systems $[17,18]$. Since 1991, GLP hens have been covered by a national program for the conservation of animal genetic resources, among the other 10 breeds/families of laying hens. This breed is also classified by the FAO as a global genetic resource [19].

The aim of this study was to assess the chemical composition (macro-, micro-, trace, and ultratrace elements) in the content of GLP hens' eggs kept in a free-range system with varied mineral nutrition. 


\section{Materials and Methods}

\subsection{Ethical Statement}

The experiment on GLP hens was approved by the Second Local Ethical Committee on Animal Testing at Wroclaw University of Environmental and Life Sciences (UPWr) (No. 17/2009, 9 February 2009).

\subsection{Animal Population and Experimental Design}

All experiments were carried out in hen farms in Modrze (Poland). The animal material consisted of 300 GLP hens, which were divided into two experimental groups (Gr-1 and Gr-2) of 150 birds each. At the beginning of the experiment, the hens were 18 weeks of age and were kept until the peak of laying (32-33 weeks). The birds were kept in a free-range system and had free access to green, partly grassy paddocks with an area of $8 \mathrm{~m}^{2} /$ head. At night, the birds stayed in henhouses equipped with feeders, drinkers, three-story perches, and nest boxes. The floor of the henhouses was covered with crushed straw and sawdust. There were 5 hens $/ \mathrm{m}^{2}$ inside the henhouse, the perch length was $25 \mathrm{~cm} / \mathrm{hen}$, and there were six hens per nest. The birds had constant access to feed and water.

\subsection{Feed Mixture}

The basal feed mixture was formulated according to the poultry nutrition standards [20]. The hens were fed with feed mixtures based on corn meal, soybean meal, postextraction rapeseed meal, wheat (grains and bran), dicalcium phosphate, ground limestone, salt and vitamin-mineral premix, with Gr-2 birds additionally receiving mineral organic feed additives (MOFA) which was $10 \%$ of the feed mixture. The feed mixture was produced under veterinary supervision on the farm where the hens were kept. The MOFA were composed of flaxseed meal, dry microalgae, dolomite, humin, and aluminosilicate raw materials. Both mixtures were sent to the laboratory at the Department of Animal Nutrition and Feed Management of UPWr. In this laboratory, the basic parameters of feed mixture were determined (crude protein, crude fat, crude fiber, crude ash, and dry matter) [21].

\subsection{Sample Collection for Analyses}

Eggs used for tests were collected at the peak of laying for five successive days from both groups. Every day, five undamaged eggs with similar weight (53-57 g) were randomly selected from each experimental groups. In the next step, the eggs were broken and the albumen and yolk were separated. Every day, five samples $(n=5)$ of albumen and yolk were collected from both groups, resulting in a total of 50 samples. Samples were stored for 6 days in a coldroom $\left(+4{ }^{\circ} \mathrm{C}\right)$ before transport to the chemical laboratory at the Wroclaw University of Science and Technology (WUST). Additionally, approximately $0.5 \mathrm{~kg}(n=5)$ samples of the feed mixtures were collected for chemical analysis at weekly intervals from the fifth week before egg collection.

\subsection{Analitical Techniques}

The chemical composition of the feed mixture and hen eggs was tested to determine chemical element content. Analyses were performed using inductively coupled plasma spectroscopy by ICP-OES and ICP-MS techniques. The appropriate mass of biological material (feed, $0.5 \mathrm{~g}$; albumen, $1.5 \mathrm{~g}$; and yolk, $1.5 \mathrm{~g}$ ) was mineralized in Teflon bombs with $5 \mathrm{~mL}$ of concentrated, spectrally pure nitric acid $\left(\mathrm{HNO}_{3}\right.$, from Merck) in a START $\mathrm{D}$ microwave digestion system (Milestone, Italy). The following analytical equipment was used: an inductively coupled plasma optical emission spectrometer with ultrasonic nebulizer (Varian VISTA-MPX ICP-OES, Melbourne, Victoria, Australia); and an inductively coupled plasma spectrometer with mass detector (ICP-MS Varian Ultra Mass-700 Instrument, Melbourne, Victoria, Australia). 
In the feed mixture and egg content (albumen and yolk), the concentration of the following elements was determined by ICP-MS method: $\mathrm{Co}, \mathrm{Cr}, \mathrm{Cu}, \mathrm{Fe}, \mathrm{I}, \mathrm{Mn}, \mathrm{Mo}, \mathrm{Se}$, and $\mathrm{Zn}$. Analysis of the remaining elements was performed by ICP-OES: macro- and microelements $\mathrm{Ca}, \mathrm{K}, \mathrm{P}, \mathrm{S}, \mathrm{Mg}$, $\mathrm{Na}$ and $\mathrm{Al}, \mathrm{Ba}, \mathrm{Si}$, Sr; trace elements $\mathrm{As}, \mathrm{B}, \mathrm{Be}, \mathrm{Bi}, \mathrm{Cd}$, $\mathrm{Cs}, \mathrm{Ga}, \mathrm{Ge}, \mathrm{Li}, \mathrm{Nd}, \mathrm{Ni}, \mathrm{Pb}, \mathrm{Rb}, \mathrm{Ti}, \mathrm{V}, \mathrm{Y}$, and $\mathrm{Zr}$; and ultratrace elements $\mathrm{Ag}, \mathrm{Au}, \mathrm{Ce}, \mathrm{Gd}$, $\mathrm{Hf}, \mathrm{La}, \mathrm{Lu}, \mathrm{Nb}, \mathrm{Pd}, \mathrm{Pt}, \mathrm{Ru}, \mathrm{Sb}, \mathrm{Sn}, \mathrm{Ta}, \mathrm{Te}, \mathrm{Tl}, \mathrm{U}, \mathrm{W}$, and $\mathrm{Yb}$. Mercury (Hg) content was analyzed by atomic absorption spectrometry, mercury analyzer AMA-254 (Altec Ltd., Prague, Czech Republic). Anions, such as $\mathrm{Cl}, \mathrm{F}, \mathrm{Br}$, and radioactive elements (except $\mathrm{U}$ ), were not determined. These elements were bonded chemically to metals, with the exception of $\mathrm{B}, \mathrm{I}, \mathrm{Se}, \mathrm{Si}$, and Te (non-metals), although elements such as Al, As, B, Ge, Sb, Se, and Te are classified as metalloids [22,23].

Analytical determinations were carried out in the Laboratory of Multielemental Analyses at WUST, accredited by the Polish Center for Accreditation and ILAC/MRA, No. AB 696. The analytical determinations were performed in accordance with PN-EN ISO 17025. The quality of analytical process was controlled with the certified reference material: whole egg powder (NIST RM 8415), corn flour (INCT-CF-3) and soya bean flour (INCT-SBF-4).

To avoid possible secondary contamination of the sample, no metal tools and laboratory equipment were used, only those made of plastic or glass.

\subsection{Statistical Analysis}

Analysis of the results was carried out using Statistica ver. 13.1. The data is presented as the mean and standard error of mean (SEM). The Shapiro-Wilk test was used to determine the normality of the data distribution. If the distribution was normal, a Student's $t$-test for independent samples was performed. If the distribution was not normal, a Mann-Whitney $U$ test was performed. Differences were considered significant if $p<0.05$ or $p<0.01$.

\section{Results}

\subsection{Feed Mixture}

The results relating to the feed mixtures are presented in Table 1 . Table 2 shows the percentage of macro- and microelements in both feed mixtures. Their chemical composition (macro-, micro-, trace, and ultratrace elements) is presented in Tables 3 and 4.

Table 1. Nutrient composition of feed mixtures for laying hens.

\begin{tabular}{ccc}
\hline Compound & Gr-1 & Gr-2 * \\
\hline Dry matter (\%) & 90.25 & 91.28 \\
Crude ash (\%) & 12.03 & 12.69 \\
Crude fiber (\%) & 6.08 & 6.71 \\
Crude fat (\%) & 3.88 & 5.02 \\
Crude protein $(\%)$ & 16.92 & 17.57 \\
Metabolic energy $(\mathrm{kcal} / \mathrm{kg})$ & 2684 & 2711 \\
\hline
\end{tabular}

${ }^{*}$ Basal diet + inclusion of 10\% MOFA (flaxseed meal, dry microalgae biomass, dolomite, humin, and aluminosilicate raw materials).

Table 2. The percentage of macro- and microelements in the feed mixture (\%).

\begin{tabular}{ccc}
\hline Element & Gr-1 & Gr-2 \\
\hline & Macroelements & \\
\hline $\mathrm{Ca}$ & 3.553 & 3.771 \\
$\mathrm{~K}$ & 0.627 & 0.683 \\
$\mathrm{P}$ & 0.476 & 0.528 \\
$\mathrm{~S}$ & 0.230 & 0.284 \\
$\mathrm{Mg}$ & 0.208 & 0.217 \\
$\mathrm{Na}$ & 0.149 & 0.157 \\
\hline
\end{tabular}


Table 2. Cont.

\begin{tabular}{ccc}
\hline Element & Gr-1 & Gr-2 \\
\hline & Microelements & \\
\hline $\mathrm{Al}$ & 0.0271 & 0.0331 \\
$\mathrm{Fe}$ & 0.0266 & 0.0302 \\
$\mathrm{Si}$ & 0.0204 & 0.0252 \\
$\mathrm{Mn}$ & 0.0112 & 0.0107 \\
$\mathrm{Zn}$ & 0.0093 & 0.0106 \\
$\mathrm{Sr}$ & 0.0019 & 0.0027 \\
$\mathrm{Cu}$ & 0.0016 & 0.0017 \\
$\mathrm{Ba}$ & 0.0003 & 0.0004 \\
$\mathrm{I}$ & 0.0003 & 0.0002 \\
\hline
\end{tabular}

Table 3. The concentrations of macro- and microelements in the feed mixture $(\mathrm{mg} / \mathrm{kg})$.

\begin{tabular}{ccccc}
\hline Element & Gr-1 & Gr-2 & SEM & $p$-Value \\
\hline \multicolumn{5}{c}{ Macroelements } \\
\hline $\mathrm{Ca}$ & $35537.0^{\mathrm{a}}$ & $37713.3^{\mathrm{b}}$ & 598.22 & $0.037^{\dagger}$ \\
$\mathrm{K}$ & $6272.4^{\mathrm{a}}$ & $6835.8^{\mathrm{b}}$ & 104.28 & $0.00013^{\dagger}$ \\
$\mathrm{P}$ & $4763.5^{\mathrm{a}}$ & $5285.2^{\mathrm{b}}$ & 126.05 & $0.027^{\dagger}$ \\
$\mathrm{S}$ & $2301.8^{\mathrm{A}}$ & $2846.4^{\mathrm{B}}$ & 297.63 & $0.00001^{\dagger}$ \\
$\mathrm{Mg}$ & 2082.0 & 2175.1 & 40.58 & $0.276^{\dagger}$ \\
$\mathrm{Na}$ & 1495.2 & 1577.4 & 34.67 & $0.258^{\dagger}$ \\
\hline \multicolumn{4}{c}{ Microelements } \\
$\mathrm{Al}$ & $271.10^{\mathrm{a}}$ & $331.41^{\mathrm{b}}$ & 15.72 & $0.046^{\dagger}$ \\
$\mathrm{Fe}$ & 266.37 & 302.34 & 12.70 & $0.168^{\dagger}$ \\
$\mathrm{Si}$ & $204.78^{\mathrm{a}}$ & $252.64^{\mathrm{b}}$ & 11.35 & $0.023^{\dagger}$ \\
$\mathrm{Mn}$ & 112.14 & 107.55 & 4.061 & $0.601^{\dagger}$ \\
$\mathrm{Zn}$ & $93.48^{\dagger}$ & 106.26 & 6.892 & $0.384^{\dagger}$ \\
$\mathrm{Sr}$ & 19.32 & 27.08 & 2.187 & $0.071^{\dagger}$ \\
$\mathrm{Cu}$ & 16.74 & 17.56 & 0.634 & $0.549^{\dagger}$ \\
$\mathrm{Ba}$ & $3.09^{\mathrm{a}}$ & $4.35^{\mathrm{b}}$ & 0.282 & $0.013^{\dagger}$ \\
$\mathrm{I}$ & 3.08 & 2.74 & 0.194 & $0.414^{\dagger}$
\end{tabular}

${ }^{\dagger}$ based on $t$-test; ${ }^{\mathrm{a}, \mathrm{b}}$ significance of differences on the level $p<0.05 ;{ }^{\mathrm{A}, \mathrm{B}}$ significance of differences on the level $p<0.01$; SEM, standard error of mean.

Table 4. The concentrations of trace and ultratrace elements in the feed mixture $(\mathrm{mg} / \mathrm{kg})$.

\begin{tabular}{ccccc}
\hline Element & Gr-1 & Gr-2 & SEM & $p$-Value \\
\hline \multicolumn{5}{c}{ Trace elements } \\
\hline $\mathrm{Ti}$ & $6.420^{\mathrm{a}}$ & $7.781^{\mathrm{b}}$ & 0.469 & $0.157^{\dagger}$ \\
$\mathrm{Cr}$ & $3.344^{\mathrm{A}}$ & $4.598^{\mathrm{B}}$ & 0.2195 & $0.0003^{\dagger}$ \\
$\mathrm{Rb}$ & $3.320^{\mathrm{A}}$ & 3.193 & 0.1772 & $0.7330^{\dagger}$ \\
$\mathrm{Ni}$ & $1.329^{\mathrm{A}}$ & $2.541^{\mathrm{B}}$ & 0.2422 & $0.0024^{\dagger}$ \\
$\mathrm{V}$ & 1.294 & 0.882 & 0.1781 & $0.2721^{\dagger}$ \\
$\mathrm{Y}$ & 1.178 & 0.989 & 0.1590 & $0.5820^{\dagger}$ \\
$\mathrm{Bi}$ & 0.866 & 0.562 & 0.1403 & $0.0681^{\dagger}$ \\
$\mathrm{Mo}$ & 0.757 & 0.853 & 0.0872 & $0.6112^{\dagger}$ \\
$\mathrm{Se}$ & 0.586 & 0.604 & 0.0951 & $0.9991^{\ddagger}$ \\
$\mathrm{Ga}$ & 0.505 & 0.396 & 0.0490 & $0.2880^{\dagger}$ \\
$\mathrm{Ce}$ & 0.448 & 0.327 & 0.0891 & $0.2959^{\ddagger}$ \\
$\mathrm{B}$ & 0.409 & 0.462 & 0.0142 & $0.0512^{\dagger}$ \\
\hline
\end{tabular}


Table 4. Cont.

\begin{tabular}{|c|c|c|c|c|}
\hline Element & Gr-1 & Gr-2 & SEM & $p$-Value \\
\hline \multicolumn{5}{|c|}{ Trace elements } \\
\hline As & 0.404 & 0.668 & 0.0309 & $0.1432 \ddagger$ \\
\hline $\mathrm{La}$ & 0.397 & 0.323 & 0.0032 & $0.3421^{\dagger}$ \\
\hline $\mathrm{Li}$ & $0.328^{\mathrm{A}}$ & $0.450^{\mathrm{B}}$ & 0.0384 & $0.0002^{+}$ \\
\hline $\mathrm{Nd}$ & 0.294 & 0.185 & 0.0331 & $0.1070^{+}$ \\
\hline $\mathrm{Zr}$ & $0.253^{\mathrm{a}}$ & $0.464^{\mathrm{b}}$ & 0.0293 & $0.0212 \ddagger$ \\
\hline $\mathrm{Pb}$ & $0.242^{\mathrm{a}}$ & $0.345^{b}$ & 0.0041 & $0.0170^{+}$ \\
\hline Co & $0.171^{\mathrm{a}}$ & $0.280^{b}$ & 0.0932 & $0.0194^{\dagger}$ \\
\hline Sn & $0.164^{\mathrm{a}}$ & $0.293^{b}$ & 0.0522 & $0.0473 \ddagger$ \\
\hline $\mathrm{Ge}$ & 0.156 & 0.106 & 0.0237 & $0.3072^{\dagger}$ \\
\hline \multicolumn{5}{|c|}{ Ultratrace elements } \\
\hline $\mathrm{Pd}$ & $0.067^{\mathrm{A}}$ & $0.041^{\mathrm{B}}$ & 0.0410 & $0.0061^{\dagger}$ \\
\hline $\mathrm{Gd}$ & 0.065 & 0.042 & 0.0013 & $0.1313^{+}$ \\
\hline $\mathrm{Cd}$ & 0.064 & 0.060 & 0.0042 & $0.7876^{+}$ \\
\hline $\mathrm{Lu}$ & 0.042 & 0.036 & 0.0002 & $0.4240^{\dagger}$ \\
\hline $\mathrm{Sb}$ & 0.041 & 0.031 & 0.0462 & $0.2911^{\dagger}$ \\
\hline $\mathrm{Nb}$ & $0.035^{\mathrm{A}}$ & $0.052^{\mathrm{B}}$ & 0.0033 & $0.0017 \ddagger$ \\
\hline $\mathrm{Be}$ & 0.035 & 0.030 & 0.0072 & $0.6141^{\dagger}$ \\
\hline $\mathrm{Yb}$ & 0.026 & 0.023 & 0.0022 & $0.3901^{+}$ \\
\hline $\mathrm{Ag}$ & 0.022 & 0.018 & 0.0021 & $0.4922^{+}$ \\
\hline Cs & $0.018^{\mathrm{a}}$ & $0.011^{b}$ & 0.0018 & $0.0211^{\dagger}$ \\
\hline $\mathrm{Hg}$ & 0.0110 & 0.0070 & 0.00694 & $0.39379^{+}$ \\
\hline U & 0.0058 & 0.0039 & 0.00121 & $0.13834^{+}$ \\
\hline W & 0.0054 & 0.0063 & 0.00103 & $0.55510^{+}$ \\
\hline $\mathrm{Te}$ & $0.0053^{a}$ & $0.0037^{b}$ & 0.00141 & $0.04513^{\dagger}$ \\
\hline Hf & 0.0038 & 0.0048 & 0.00703 & $0.54331^{\dagger}$ \\
\hline $\mathrm{Ru}$ & $0.0034^{a}$ & $0.0073^{b}$ & 0.00248 & $0.04248^{+}$ \\
\hline $\mathrm{Au}$ & 0.0016 & 0.0017 & 0.00022 & $0.84021^{\dagger}$ \\
\hline $\mathrm{Pt}$ & 0.0015 & 0.0020 & 0.00330 & $0.54223^{\dagger}$ \\
\hline $\mathrm{Tl}$ & 0.0014 & 0.0012 & 0.00012 & $0.51851^{\dagger}$ \\
\hline $\mathrm{Ta}$ & 0.00066 & 0.00061 & 0.00114 & $0.51252^{+}$ \\
\hline
\end{tabular}

${ }^{\dagger}$ based on Mann-Whitney $U$ test; ${ }^{\mathrm{a}, \mathrm{b}}$ significance of differences on the level $p<0.05$; ${ }^{\mathrm{A}, \mathrm{B}}$ significance of differences on the level $p<0.01$; SEM standard error of mean.

The total macro- and microelement content was $53,466.0 \mathrm{mg} / \mathrm{kg}$ in $\mathrm{Gr}-1$ and $57,411.8 \mathrm{mg} / \mathrm{kg}$ in Gr-2 $(\Delta=7.38 \%)$. In contrast, trace and ultratrace element content was 23.32 and $26.68 \mathrm{mg} / \mathrm{kg}$, respectively $(\Delta=12.59 \%)$.

Among 56 elements, significant differences were observed for macro- and microelements. In Gr-2 feed mixture, the level of $\mathrm{Al}, \mathrm{Ba}, \mathrm{Ca}, \mathrm{K}, \mathrm{P}, \mathrm{S}$, and Si was significantly higher $(p<0.05$ or $p<0.01)$. Ca and $\mathrm{K}$ were the most abundant in the feed mixtures, whereas $\mathrm{Ba}$ and I levels were the lowest. Among the trace and ultratrace elements, higher levels in $\mathrm{Gr}-2$ feed mixture of the following elements were determined: $\mathrm{Co}, \mathrm{Cr}, \mathrm{Li}, \mathrm{Nb}, \mathrm{Ni}, \mathrm{Ru}, \mathrm{Sn}$, and $\mathrm{Zr}$, and lower levels of $\mathrm{Cs}, \mathrm{Pd}$ and Te. Among trace and ultratrace elements, $\mathrm{Cr}, \mathrm{Rb}$, and $\mathrm{Ti}$ were the most abundant in the feed mixtures, while $\mathrm{Au}, \mathrm{Pt}, \mathrm{Ta}$, and $\mathrm{Tl}$ were below $0.02 \mathrm{mg} / \mathrm{kg}$.

\subsection{Egg Albumen}

Tables 5 and 6 summarize the results of the albumen analyses. Among the examined elements in the group of macro- and microelements, significant differences were observed for $\mathrm{Ba}$, and higher levels were found in $\mathrm{Gr}-2$. Na and $\mathrm{S}$ were the most abundant in albumen, and $\mathrm{Ba}$ and $\mathrm{Mn}$ the least. 
Table 5. The concentrations of macro- and microelements in the egg albumen (mg/kg).

\begin{tabular}{ccccc}
\hline Element & Gr-1 & Gr-2 & SEM & $p$-Value \\
\hline \multicolumn{4}{c}{ Macroelements } \\
\hline $\mathrm{S}$ & 1981.2 & 2001.0 & 25.91 & $0.834^{\ddagger}$ \\
$\mathrm{Na}$ & 1926.3 & 2008.2 & 26.17 & $0.121^{\dagger}$ \\
$\mathrm{K}$ & 1480.4 & 1430.3 & 20.18 & $0.233^{\dagger}$ \\
$\mathrm{Mg}$ & 124.00 & 119.85 & 4.113 & $0.638^{\dagger}$ \\
$\mathrm{P}$ & 111.78 & 104.70 & 3.101 & $0.278^{\dagger}$ \\
$\mathrm{Ca}$ & 103.16 & 104.88 & 3.649 & $0.829^{\dagger}$ \\
\hline & Microelements & \\
\hline $\mathrm{Si}$ & 7.184 & 6.475 & 0.3192 & $0.2863^{\dagger}$ \\
$\mathrm{Fe}$ & 0.590 & 0.624 & 0.0241 & $0.6311^{\dagger}$ \\
$\mathrm{Al}$ & 0.532 & 0.579 & 0.0232 & $0.3372^{\dagger}$ \\
$\mathrm{Cu}$ & 0.258 & 0.230 & 0.0246 & $0.6009^{\dagger}$ \\
$\mathrm{Zn}$ & 0.187 & 0.223 & 0.0092 & $0.2062^{\dagger}$ \\
$\mathrm{Sr}$ & 0.143 & 0.136 & 0.0050 & $0.3930^{\dagger}$ \\
$\mathrm{I}$ & 0.062 & 0.076 & 0.0115 & $0.5770^{\dagger}$ \\
$\mathrm{Ba}$ & $0.055^{\mathrm{a}}$ & $0.087^{\mathrm{b}}$ & 0.0075 & $0.0122^{\ddagger}$ \\
$\mathrm{Mn}$ & 0.018 & 0.012 & 0.0026 & $0.2797^{\dagger}$ \\
\hline
\end{tabular}

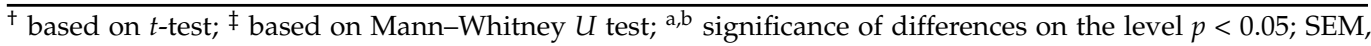
standard error of mean.

Table 6. The concentrations of trace and ultratrace elements in the egg albumen $(\mathrm{mg} / \mathrm{kg})$.

\begin{tabular}{|c|c|c|c|c|}
\hline Element & Gr-1 & Gr-2 & SEM & $p$-Value \\
\hline \multicolumn{5}{|c|}{ Trace elements } \\
\hline $\mathrm{Rb}$ & 1.098 & 1.323 & 0.1192 & $0.2091^{\dagger}$ \\
\hline $\mathrm{Zr}$ & 0.632 & 0.575 & 0.0083 & $0.8390^{+}$ \\
\hline $\mathrm{Ti}$ & 0.241 & 0.287 & 0.0375 & $0.6544^{+}$ \\
\hline $\mathrm{V}$ & 0.203 & 0.160 & 0.0155 & $0.1953^{+}$ \\
\hline $\mathrm{B}$ & 0.159 & 0.154 & 0.0073 & $0.7841^{+}$ \\
\hline Mo & $0.154^{\mathrm{A}}$ & $0.247^{\mathrm{B}}$ & 0.0352 & $0.0011^{+}$ \\
\hline Se & $0.121^{a}$ & $0.142^{b}$ & 0.0042 & $0.0410^{+}$ \\
\hline $\mathrm{Ag}$ & 0.081 & 0.083 & 0.0021 & $0.5699^{\dagger}$ \\
\hline $\mathrm{Ce}$ & 0.067 & 0.062 & 0.0094 & $0.6761^{\ddagger}$ \\
\hline $\mathrm{Cr}$ & $0.066^{\mathrm{A}}$ & $0.109^{\mathrm{B}}$ & 0.0132 & $0.0002^{+}$ \\
\hline As & $0.053^{\mathrm{A}}$ & $0.026^{\mathrm{B}}$ & 0.0053 & $0.0027^{+}$ \\
\hline $\mathrm{Ni}$ & 0.021 & 0.026 & 0.0027 & $0.3245^{\dagger}$ \\
\hline $\mathrm{Bi}$ & 0.018 & 0.022 & 0.0013 & $0.0738^{+}$ \\
\hline $\mathrm{Ga}$ & 0.017 & 0.018 & 0.0004 & $0.5434^{+}$ \\
\hline $\mathrm{Pd}$ & 0.015 & 0.011 & 0.0055 & $0.2076^{+}$ \\
\hline $\mathrm{Pb}$ & 0.012 & 0.015 & 0.0009 & $0.4263^{+}$ \\
\hline $\mathrm{Ge}$ & 0.013 & 0.014 & 0.0008 & $0.7516^{\dagger}$ \\
\hline \multicolumn{5}{|c|}{ Ultratrace elements } \\
\hline $\mathrm{Sb}$ & 0.0092 & 0.0112 & 0.00336 & $0.29627 \ddagger$ \\
\hline $\mathrm{Li}$ & 0.0090 & 0.0102 & 0.00101 & $0.58465^{\dagger}$ \\
\hline Sn & 0.0084 & 0.0088 & 0.00034 & $0.55455^{\dagger}$ \\
\hline $\mathrm{Te}$ & 0.0080 & 0.0121 & 0.00212 & $0.83453 \ddagger$ \\
\hline $\mathrm{Be}$ & 0.0066 & 0.0048 & 0.00050 & $0.06658^{\dagger}$ \\
\hline $\mathrm{Lu}$ & 0.0061 & 0.0082 & 0.00061 & $0.07797^{\dagger}$ \\
\hline Co & $0.0048^{a}$ & $0.0074^{b}$ & 0.00058 & $0.01379^{\dagger}$ \\
\hline $\mathrm{Ru}$ & $0.0044^{\mathrm{A}}$ & $0.0083^{B}$ & 0.00094 & $0.00168^{\dagger}$ \\
\hline $\mathrm{Y}$ & 0.0036 & 0.0041 & 0.00055 & $0.72964^{\dagger}$ \\
\hline $\mathrm{La}$ & 0.0024 & 0.0035 & 0.00044 & $0.26402^{\dagger}$ \\
\hline $\mathrm{Au}$ & $0.0021^{\mathrm{A}}$ & $0.0048^{B}$ & 0.00057 & $0.00483^{\dagger}$ \\
\hline
\end{tabular}


Table 6. Cont.

\begin{tabular}{ccccc}
\hline Element & Gr-1 & Gr-2 & SEM & $p$-Value \\
\hline \multicolumn{5}{c}{ Ultratrace elements } \\
\hline $\mathrm{Nd}$ & 0.0017 & 0.0014 & 0.00011 & $0.38923^{\dagger}$ \\
$\mathrm{Nb}$ & $0.0014^{\mathrm{A}}$ & $0.0029^{\mathrm{B}}$ & 0.00048 & $0.00062^{\dagger}$ \\
$\mathrm{Yb}$ & 0.0011 & 0.0012 & 0.00017 & $0.46470^{\ddagger}$ \\
$\mathrm{Tl}$ & 0.00108 & 0.00090 & 0.000163 & $0.612099^{\dagger}$ \\
$\mathrm{Pt}$ & 0.00102 & 0.00132 & 0.000460 & $0.428910^{\dagger}$ \\
$\mathrm{Gd}$ & 0.00091 & 0.00104 & 0.000122 & $0.650436^{\dagger}$ \\
$\mathrm{W}$ & 0.00088 & 0.00083 & 0.000084 & $0.778010^{\dagger}$ \\
$\mathrm{Cd}$ & 0.00083 & 0.00140 & 0.000250 & $0.270439^{\dagger}$ \\
$\mathrm{Cs}$ & 0.00066 & 0.00054 & 0.000049 & $0.246250^{\dagger}$ \\
$\mathrm{Hg}$ & 0.00058 & 0.00066 & 0.000035 & $0.250634^{\dagger}$ \\
$\mathrm{Hf}$ & 0.00050 & 0.00060 & 0.000088 & $0.588286^{\dagger}$ \\
$\mathrm{Ta}$ & 0.00022 & 0.00025 & 0.000022 & $0.566521^{\dagger}$ \\
$\mathrm{U}$ & 0.00018 & 0.00021 & 0.000021 & $0.631920^{\dagger}$ \\
\hline
\end{tabular}

${ }^{\dagger}$ based on $t$-test; ${ }^{\ddagger}$ based on Mann-Whitney $U$ test; ${ }^{a, b}$ significance of differences on the level $p<0.05$; $\mathrm{A}, \mathrm{B}$ significance of differences on the level $p<0.01$; SEM, standard error of mean.

Among the trace and ultratrace elements, there were significantly higher concentrations of the following elements in $\mathrm{Gr}-2 \mathrm{~A} \mathrm{Au}, \mathrm{Co}, \mathrm{Cr}, \mathrm{Mo}, \mathrm{Nb}, \mathrm{Ru}$, and Se, and lower levels of only As in Gr-1. The most abundant trace and ultratrace elements in the albumen were (in descending order) $\mathrm{Rb}, \mathrm{Ti}$, and $\mathrm{Zr}$, while the least—-below $0.001 \mathrm{mg} / \mathrm{kg}$ - was $\mathrm{Cd}, \mathrm{Cs}, \mathrm{Gd}$, $\mathrm{Hf}, \mathrm{Hg}, \mathrm{Ta}, \mathrm{U}$, and $\mathrm{W}$.

The total macro- and microelement content was $5735.8 \mathrm{mg} / \mathrm{kg}$ in Gr-1 and $5777.1 \mathrm{mg} / \mathrm{kg}$ in Gr-2 $(\Delta=0.72 \%)$, while trace and ultratrace element content was 3.046 and $3.372 \mathrm{mg} / \mathrm{kg}$, respectively $(\Delta=10.70 \%)$.

\subsection{Egg Yolk}

Tables 7 and 8 summarize the results of the yolk analyses. Among 15 macro- and microelements, no significant differences were found between $\mathrm{Gr}-1$ and $\mathrm{Gr}-2$. The higher level of $\mathrm{Ca}, \mathrm{P}$, and $\mathrm{S}$ was detected in the yolk, and the lower of $\mathrm{Ba}, \mathrm{Mn}$, and $\mathrm{Sr}$.

Table 7. The concentrations of macro- and microelements in the egg yolk $(\mathrm{mg} / \mathrm{kg})$.

\begin{tabular}{ccccc}
\hline Element & Gr-1 & Gr-2 & SEM & $p$-Value \\
\hline \multicolumn{5}{c}{ Macroelements } \\
\hline $\mathrm{P}$ & 6326.2 & 6541.8 & 609.57 & $0.606^{\dagger}$ \\
$\mathrm{Ca}$ & 1838.4 & 1851.9 & 143.00 & $0.892^{\dagger}$ \\
$\mathrm{K}$ & 1513.6 & 1455.2 & 152.49 & $0.576^{\dagger}$ \\
$\mathrm{Na}$ & 1168.2 & 1202.7 & 99.342 & $0.611^{\dagger}$ \\
$\mathrm{Mg}$ & 620.10 & 643.81 & 46.753 & $0.453^{\dagger}$ \\
& 131.78 & 129.30 & 10.502 & $0.731^{\dagger}$ \\
\hline $\mathrm{Fe}$ & Microelements & & \\
$\mathrm{Zn}$ & 70.42 & 69.64 & 6.828 & $0.868^{\dagger}$ \\
$\mathrm{Si}$ & 44.51 & 39.15 & 5.060 & $0.092^{\dagger}$ \\
$\mathrm{Al}$ & 11.32 & 10.49 & 1.740 & $0.683^{\dagger}$ \\
$\mathrm{Cu}$ & 3.068 & 2.808 & 0.2599 & $0.5253^{\dagger}$ \\
$\mathrm{I}$ & 1.370 & 1.524 & 0.1122 & $0.1365^{\dagger}$ \\
$\mathrm{Mn}$ & 1.138 & 1.366 & 0.0752 & $0.4647^{\dagger}$ \\
$\mathrm{Ba}$ & 0.822 & 0.974 & 0.0936 & $0.6884^{\dagger}$ \\
$\mathrm{Sr}$ & 0.790 & 0.837 & 0.0554 & $0.5699^{\dagger}$ \\
\hline
\end{tabular}

${ }^{\dagger}$ based on $t$-test; ${ }^{\ddagger}$ based on Mann-Whitney $U$ test; SEM, standard error of mean. 
Table 8. The content of trace and ultratrace elements in the egg yolk $(\mathrm{mg} / \mathrm{kg})$.

\begin{tabular}{|c|c|c|c|c|}
\hline Element & Gr-1 & Gr-2 & SEM & $p$-Value \\
\hline \multicolumn{5}{|c|}{ Trace elements } \\
\hline $\mathrm{Rb}$ & $0.853^{a}$ & $0.629^{b}$ & 0.0552 & $0.0321^{\dagger}$ \\
\hline Se & $0.327^{\mathrm{A}}$ & $0.429^{\mathrm{B}}$ & 0.0226 & $0.0001^{+}$ \\
\hline $\mathrm{Cr}$ & $0.293^{\mathrm{A}}$ & $0.493^{\mathrm{B}}$ & 0.0418 & $0.0025^{\dagger}$ \\
\hline $\mathrm{B}$ & 0.236 & 0.256 & 0.0101 & $0.3532^{\dagger}$ \\
\hline $\mathrm{Pb}$ & 0.138 & 0.095 & 0.0182 & $0.2573^{\dagger}$ \\
\hline V & 0.153 & 0.199 & 0.0150 & $0.1358^{\dagger}$ \\
\hline Mo & 0.112 & 0.091 & 0.0105 & $0.3138^{+}$ \\
\hline $\mathrm{Ga}$ & 0.106 & 0.120 & 0.0055 & $0.2315^{\dagger}$ \\
\hline $\mathrm{Ni}$ & $0.059 \mathrm{~A}$ & $0.089^{\mathrm{B}}$ & 0.0064 & $0.0048^{+}$ \\
\hline $\mathrm{Li}$ & 0.066 & 0.055 & 0.0036 & $0.1264^{\dagger}$ \\
\hline $\mathrm{Bi}$ & 0.064 & 0.079 & 0.0069 & $0.3007^{\dagger}$ \\
\hline $\mathrm{Co}$ & $0.058^{a}$ & $0.079^{b}$ & 0.0052 & $0.0412^{+}$ \\
\hline Sn & $0.048^{\mathrm{A}}$ & $0.133^{\mathrm{B}}$ & 0.0148 & $0.0001^{\dagger}$ \\
\hline $\mathrm{Ge}$ & 0.043 & 0.049 & 0.0039 & $0.4609^{+}$ \\
\hline $\mathrm{Ti}$ & 0.039 & 0.031 & 0.0023 & $0.1038^{+}$ \\
\hline $\mathrm{Lu}$ & 0.036 & 0.032 & 0.0022 & $0.4245^{\dagger}$ \\
\hline $\mathrm{Zr}$ & 0.026 & 0.023 & 0.0015 & $0.4521^{\dagger}$ \\
\hline $\mathrm{Te}$ & 0.023 & 0.014 & 0.0025 & $0.1055^{\dagger}$ \\
\hline $\mathrm{Ag}$ & 0.022 & 0.026 & 0.0018 & $0.3462^{+}$ \\
\hline \multicolumn{5}{|c|}{ Ultratrace elements } \\
\hline $\mathrm{Au}$ & $0.0053^{a}$ & $0.0034^{b}$ & 0.00044 & $0.02124^{\dagger}$ \\
\hline $\mathrm{Hg}$ & $0.0032^{A}$ & $0.0019^{\mathrm{B}}$ & 0.00025 & $0.00541^{+}$ \\
\hline Y & $0.0048^{\mathrm{A}}$ & $0.0074^{\mathrm{B}}$ & 0.00059 & $0.02233^{\dagger}$ \\
\hline $\mathrm{Pd}$ & 0.0045 & 0.0036 & 0.00023 & $0.07406^{+}$ \\
\hline $\mathrm{Sb}$ & 0.0043 & 0.0035 & 0.00021 & $0.11011^{\dagger}$ \\
\hline $\mathrm{Nb}$ & $0.0042^{a}$ & $0.0028^{b}$ & 0.00032 & $0.01657^{\dagger}$ \\
\hline $\mathrm{Hf}$ & $0.0037^{a}$ & $0.0019^{b}$ & 0.00045 & $0.03672^{\dagger}$ \\
\hline $\mathrm{Ru}$ & 0.0033 & 0.0030 & 0.00028 & $0.59865^{+}$ \\
\hline $\mathrm{Nd}$ & $0.0032^{a}$ & $0.0020^{b}$ & 0.00029 & $0.03227^{\dagger}$ \\
\hline $\mathrm{Ce}$ & 0.0029 & 0.0036 & 0.00022 & $0.11890^{\dagger}$ \\
\hline $\mathrm{Pt}$ & 0.0027 & 0.0025 & 0.00027 & $0.76042^{\dagger}$ \\
\hline $\mathrm{La}$ & 0.0026 & 0.0024 & 0.00025 & $0.74088^{\dagger}$ \\
\hline $\mathrm{Be}$ & 0.0024 & 0.0053 & 0.00133 & $0.13815^{+}$ \\
\hline As & 0.0023 & 0.0028 & 0.00024 & $0.39135^{\dagger}$ \\
\hline $\mathrm{Gd}$ & 0.0018 & 0.0014 & 0.00013 & $0.18601^{\dagger}$ \\
\hline $\mathrm{Yb}$ & 0.0017 & 0.0019 & 0.00018 & $0.84522^{+}$ \\
\hline $\mathrm{W}$ & 0.00126 & 0.00096 & 0.000097 & $0.127859^{+}$ \\
\hline $\mathrm{Cd}$ & 0.00097 & 0.00112 & 0.000100 & $0.457444^{+}$ \\
\hline $\mathrm{Ta}$ & 0.00064 & 0.00062 & 0.000041 & $0.838300^{+}$ \\
\hline $\mathrm{Tl}$ & 0.00050 & 0.00043 & 0.000072 & $0.701493^{+}$ \\
\hline $\mathrm{U}$ & 0.00046 & 0.00044 & 0.000081 & $0.999460^{+}$ \\
\hline Cs & 0.00044 & 0.00047 & 0.000064 & $0.885814^{\dagger}$ \\
\hline
\end{tabular}

${ }^{\dagger}$ based on $t$-test; ${ }^{\mathrm{a}, \mathrm{b}}$ significance of differences on the level $p<0.05 ;{ }^{\mathrm{A}, \mathrm{B}}$ significance of differences on the level $p<0.01$; SEM, standard error of mean.

Among the studied trace and ultratrace elements, large variations occurred between the groups of elements: $\mathrm{Co}, \mathrm{Cr}, \mathrm{Ni}, \mathrm{Se}, \mathrm{Sn}$, and $\mathrm{Y}$ (more in $\mathrm{Gr}-2$ ), and $\mathrm{Au}, \mathrm{Hf}, \mathrm{Hg}, \mathrm{Nb}, \mathrm{Nd}$, and $\mathrm{Rb}$ (less in $\mathrm{Gr}-2$ ). The most abundant trace elements in the yolk were $\mathrm{Rb}$, Se, and $\mathrm{Cr}$, while those below $0.002 \mathrm{mg} / \mathrm{kg}$ were $\mathrm{Cd}, \mathrm{Cs}, \mathrm{Gd}, \mathrm{Ta}, \mathrm{Tl}, \mathrm{Yb}, \mathrm{U}$, and $\mathrm{W}$.

The total macro- and microelement content was $11732.4 \mathrm{mg} / \mathrm{kg}$ in Gr-1 and $11952.2 \mathrm{mg} / \mathrm{kg}$ in Gr-2 $(\Delta=1.87 \%)$. In contrast, trace and ultratrace elements were 2.760 and $2.977 \mathrm{mg} / \mathrm{kg}$, respectively $(\Delta=7.86 \%)$. 


\section{Discussion}

Feed mixture from Gr-2 contained the addition of MOFA, with a slightly higher (by $7.42 \%$ ) weight of minerals (macro-, micro-, trace, and ultratrace elements) compared with Gr-1. This was explained by the increase in dry mater and crude ash in the Gr-2 feed mixture. The diet used in the feeding of GLP hens differed in the content of 18 chemical elements (among 56 assessed) due to the effect of the addition of MOFA. This additive contains flaxseed, microalgae, dolomite, humin, and aluminosilicate raw materials, rich in many elements, such as $\mathrm{Al}, \mathrm{Ba}, \mathrm{Ca}, \mathrm{Co}, \mathrm{Cr}, \mathrm{Fe}, \mathrm{Ni}, \mathrm{P}, \mathrm{Si}, \mathrm{Sr}$, and $\mathrm{Ti}$ [12,24-28]. These differences generally did not affect the mineral composition of the egg albumen and yolk; only in the case of increased content of $\mathrm{Co}$ and $\mathrm{Cr}$ in $\mathrm{Gr}-2$ feed mixture, where a significant increase in these elements was found in the egg albumen and yolk of Gr-2. Similarly, an increase in $\mathrm{Ba}, \mathrm{Nb}, \mathrm{Ni}, \mathrm{Ru}$, and $\mathrm{Sn}$ in the hens' diet caused an increase in the egg albumen $(\mathrm{Ba}, \mathrm{Nb}, \mathrm{Ru})$ and yolk (Ni and $\mathrm{Sn})$.

Previous reports [12] have used HKW and HBF preparations in LB hen nutrition (ad libitum administration). The dietary humin preparations only significantly increased Se and decreased Mo concentration in the albumen, and increased Fe and Se concentrations in the yolk. These additives did not influence $\mathrm{Cr}, \mathrm{Co}, \mathrm{Cu}, \mathrm{I}, \mathrm{Mn}$, or $\mathrm{Zn}$ concentrations in the eggs. These results only partially resemble those obtained in our study; for instance, they confirmed only the increase in Fe concentration in the yolk and the lack of influence of $\mathrm{Cu}$ and $\mathrm{Zn}$ content in the albumen and yolk [12]. Other studies [29] have shown that the addition of $\mathrm{Fe}$ to the diet of laying hens, also in the organic form, does not increase its concentration in egg yolk. In contrast, I, added in organic form (yeast) to the feed of hens, significantly increased its concentration in the egg yolk [30].

It was reported [31] that the diet of laying hens can be supplemented with soybean meal enriched with $\mathrm{Cr}, \mathrm{Cu}, \mathrm{Fe}$ and $\mathrm{Zn}$. The results showed that the use of enriched soybean meal in the diet of laying hens (Hy-Line Brown) influenced the transfer of microelements to eggs, particularly at high doses. Eggs were biofortified with $\mathrm{Cu}, \mathrm{Fe}$ and $\mathrm{Zn}$ and, to a lesser extent, with $\mathrm{Cr}$. On the basis of these results, it can be concluded that the transfer of trace elements to eggs was not linearly dependent on the dose of biologically bound microelements in the diet. This proves a specific mineral independence of eggs, indicating the genetic determinants of their chemical composition.

The literature describes the physicochemical quality parameters of GLP eggs. It is known that egg albumen and yolk from these hens have a favorable amino acid composition, fatty acid profile, and higher vitamin content, mainly $\mathrm{A}$ and $\mathrm{E}$, compared with farmed eggs [32-35]. However, there is no data on the mineral composition of these eggs, especially since these birds are kept only in an extensive system, having free access to soil and vegetation of the enclosures, allowing them to eat insects and worms $[4,36]$. Only Szablewski et al. [37] provided the content of nine macro- and microelements $(\mathrm{Ca}, \mathrm{Cu}$, $\mathrm{Fe}, \mathrm{Mg}, \mathrm{Mn}, \mathrm{Na}, \mathrm{K}$, Se, and $\mathrm{Zn}$ ) in GLP eggs from hens maintained in an organic system. The results were difficult to compare, as the authors provided the content of elements for the whole egg, i.e., protein and yolk combined. In general, the mean values of these elements were similar (except for $\mathrm{Zn}$ ) to the results of the tests of eggs of other hen breeds (Sussex, RIR).

Our own research showed that the content of the main minerals and trace elements differs significantly from the standard values provided, for example by Rehault-Godbert [3]. Hence, the egg albumen of GLP had much higher levels of $\mathrm{Ca}$, I, Na, but less K, Mn, P, Se, and $\mathrm{Zn}$. The values of $\mathrm{Cu}, \mathrm{Fe}$, and $\mathrm{Mg}$ were similar. In the egg yolk of these hens, there was approximately twice as much $\mathrm{Cu}, \mathrm{Fe}, \mathrm{Mg}, \mathrm{Mn}, \mathrm{P}$, and $\mathrm{Zn}$, slightly more (by 10-20\%) $\mathrm{Ca}, \mathrm{Na}$, and $\mathrm{K}$, and less I and Se compared with the standard values [3].

The obtained results in the field of trace elements can be compared those by Giannenas et al. [14], who specified 12 trace elements in eggs from hens kept in the different systems. Similarly in the egg albumen of GLP, there was much more As, Co, Cr, $\mathrm{Mo}, \mathrm{V}$, and Se, and less $\mathrm{Mn}, \mathrm{Ni}$, and $\mathrm{Zn}$ compared with the results of the cited authors. The concentrations of $\mathrm{Cd}, \mathrm{Cu}$, and $\mathrm{Tl}$ were similar. $\mathrm{Co}, \mathrm{Cr}, \mathrm{V}$, and $\mathrm{Zn}$ concentrations in the yolk 
were higher, but As, Mo, and $\mathrm{Tl}$ were lower compared with our results (mean values of $\mathrm{Gr}-1$ and $\mathrm{Gr}-2$ ). The results of determined $\mathrm{Cd}, \mathrm{Cu}, \mathrm{Mn}, \mathrm{Ni}$, and Se concentrations were similar [14].

The obtained results can be compared with those of the tests (38 elements) carried out on the eggs of LB battery-raised hens fed with a standard feed mixture [6]. In the case of GLP in the egg albumen, the concentration of $\mathrm{Ca}, \mathrm{Cu}, \mathrm{Fe}$, and $\mathrm{Na}$ was much higher among macro- and microelements, while $\mathrm{Al}$ and $\mathrm{K}$ was lower. Among the trace elements in the egg albumen of GLP there were at least double $\mathrm{Cr}, \mathrm{Mo}, \mathrm{V}, \mathrm{Sn}, \mathrm{Ti}$, and $\mathrm{Zr}$, and lower levels of $\mathrm{Bi}$ and $\mathrm{Ni}$. In the case of the yolk, higher concentrations of $\mathrm{Ca}, \mathrm{Mn}, \mathrm{P}, \mathrm{Si}$, and $\mathrm{Sr}$, and two- or three-times higher concentrations of $\mathrm{Bi}, \mathrm{Co}$, and $\mathrm{Li}$ were observed, with much lower concentrations of $\mathrm{Ag}$, $\mathrm{As}, \mathrm{Be}, \mathrm{Ni}, \mathrm{Tl}, \mathrm{Sb}$, and $\mathrm{W}$ [6]. These differences probably stem from genetic differences and the system of maintenance. GLP hens had free access to the enclosures and could supplement their diet by eating soil, grass, insects, etc. Furthermore, in the egg albumen and yolk of Gr-2, the mass of the analyzed elements was slightly higher than in Gr-1 (by $0.90 \%$ and $1.88 \%$, respectively). The lack of testing of the water content of these edible elements of the egg made it impossible to explain this phenomenon.

In general, it seems that the eggs of GLP are characterized by higher content of many essential elements (macro- and microelements), while the concentrations of toxic elements (including heavy metals/metalloids) did not differ from the values given in previous reports [38-40]. This is of particular importance in the context of consumer health and safety. Especially that toxic metals enter the human organism mainly through the respiratory and digestive tracts. The circulation of these metals in ecosystems is primarily related to the food chains of plants, animals, and humans [41,42]. This is due to the fact that heavy metals from soil, along with water, are taken up by plants and accumulate in their tissues (mainly roots and leaves), which are then eaten by animals and humans [43,44]. It should also be remembered that the amount of absorbed metal depends on its form, $\mathrm{pH}$ in the digestive tract, the speed of its passage through the digestive system, and the presence of other substances that may modify its absorption. However, the interpretation of the results is complicated due to the complex relationships between chemical elements (synergistic, antagonistic, and agonistic), which makes it difficult to interpret the results objectively, even with the appropriate statistical analysis performed $[40,45,46]$.

\section{Conclusions}

The results of the chemical analyses show that the mineral composition of feed mixtures has a slight effect on the mean values of macro-, micro-, trace, and ultratrace elements in the content of GLP eggs. Only in the case of increased Co and Cr content, a significant increase in their concentration in egg albumen and yolk was found (Gr-2). Similarly, a significant increase in $\mathrm{Ba}, \mathrm{Nb}$, and $\mathrm{Ru}$ in the diet of hens causes growth in the egg albumen, and, in the case of $\mathrm{Ni}$ and $\mathrm{Sn}$, in the egg yolk. This proves the specific mineral independence of eggs, indicating the genetic determinants of their chemical composition. The possible enrichment of the content of consumed eggs with bioactive minerals through the alimentary way (biofortification) may be problematic from a physiological and practical point of view.

Author Contributions: Conceptualization, Z.D. and T.T.; methodology, T.T., Z.D. and K.C.; software, D.K. and A.B.; validation, D.K. and A.B.; formal analysis, K.C. and M.K.; investigation, M.K. and W.T.; resources, M.K. and W.T.; data curation, A.B. and H.B.; writing-original draft preparation, Z.D. and A.B.; writing-review and editing, Z.D. and K.C.; visualization, D.K.; supervision, T.T. and K.C.; project administration, A.B. and H.B.; and funding acquisition, T.T. All authors have read and agreed to the published version of the manuscript.

Funding: Performed during the realization of project No. POIG.01.03.01-00-133/08.

Institutional Review Board Statement: Not applicable.

Informed Consent Statement: Not applicable. 
Data Availability Statement: The data presented in this study are available on request from the corresponding author.

Acknowledgments: This study was funded by the project "Innovative technologies of production of biopreparations based on new generation eggs (OVOCURA)". The project is co-financed by the European Regional Development Fund within the Innovative Economy 2007-2014 Operational Programme-Wroclaw University of Environmental and Life Sciences. The article processing charge is financed under the Leading Research Groups support project from the subsidy increased for the period 2020-2025 in the amount of $2 \%$ of the subsidy referred to in Art. 387 (3) of the Law of 20 July 2018 on Higher Education and Science, obtained in 2019.

Conflicts of Interest: The authors declare no conflict of interest.

\section{References}

1. Herron, K.L.; Fernandez, M.L. Are the current dietary guidelines regarding egg composition appropriate? J. Nutr. 2004, 134, 187-190. [CrossRef]

2. Trziszka, T.; Polanowski, A.; Szuba, A.; Rymaszewska, J.; Dobrzański, Z. Eggs as a source of nutraceuticals and biomedical substances in the prevention of civilization diseases. World's Poult. Sci. 2013, 69, 75-78.

3. Réhault-Godbert, S.; Guyot, N.; Nys, Y. The Golden Egg: Nutritional Value, Bioactivities, and Emerging Benefits for Human Health. Nutrients 2019, 11, 684. [CrossRef]

4. Karsten, H.D.; Patterson, P.H.; Stout, R.; Crews, G. Vitamins A, E and fatty acid composition of the eggs of caged hens and pastured hens. Renew. Agric. Food Syst. 2010, 25, 45-54. [CrossRef]

5. Kiczorowska, B.; Samolińska, W.; Kwiecień, M.; Winiarska-Mieczan, A.; Rusinek-Prystupa, E.; Al-Yasiry, A.R.M. Nutritional value and the content of minerals in eggs produced in large-scale, courtyard and organic systems. J. Elem. 2015, 20, 887-895. [CrossRef]

6. Dobrzański, Z.; Chojnacka, K.; Trziszka, T.; Opaliński, S.; Bobak, Ł.; Konkol, D.; Korczyński, M. The effect of dietary humic preparations on the content of essential and non-essential chemical elements in hen eggs. Animals 2020, 10, 1252. [CrossRef] [PubMed]

7. Noel, L.; Chekri, R.; Millour, S.; Vastel, C.; Kadar, A.; Sirot, V.; Leblanc, J.C.; Guerin, T. Li, Cr, Mn, Co, Ni, Cu, Zn, Se, and Mo levels in foodstuffs from the Second French TDS. Food Chem. 2012, 132, 1502-1513. [CrossRef] [PubMed]

8. Chellan, P.; Sadler, P.J. The elements of life and medicines. Philos. Trans. A Math. Phys. Eng. Sci. 2015, 373, 20140182. [CrossRef] [PubMed]

9. Zoroddu, M.A.; Aashet, J.; Crisponi, G.; Medici, S.; Peana, M.; Nurchi, V.M. The essential metals for humans: A brief overview. J. Inorg. Biochem. 2019, 195, 120-129. [CrossRef] [PubMed]

10. Hashish, S.M.; Abdel-Samee, L.D.; Abdel-Wahhab, M.A. Mineral and Heavy Metals Content in Eggs of Local Hens at Different Geographic Areas in Egypt. Glob. Vet. 2012, 8, 298-304.

11. Kucukyilmaz, K.; Bozkurt, M.; Yamaner, C.; Cinar, M.; Catli, A.U.; Konak, R. Effect of an organic and conventional rearing system on the mineral content of hen eggs. Food Chem. 2012, 132, 989-992. [CrossRef]

12. Dobrzański, Z.; Górecki, H.; Chojnacka, K.; Górecka, H.; Synowiec, M. Effect of dietary humic preparations on the content of the trace elements in hens' eggs. Am. J. Agric. Biol. Sci. 2007, 2, 234-240.

13. Vincevica-Gaile, Z.; Gaga, K.; Klavins, M. Food and environment: Trace element content of hen eggs from different housing types. APCBEE Procedia 2013, 5, 221-226. [CrossRef]

14. Giannenas, I.; Nisianakis, P.; Gavriil, A.; Kontopidis, G.; Kyriazakis, I. Trace mineral content of conventional, organic and courtyard eggs analysed by inductively coupled plasma mass spectrometry (ICP-MS). Food Chem. 2009, 114, 706-711. [CrossRef]

15. Salwa, A.A. Determination of some trace elements in chicken eggs from different sources. J. Pharmacogn. Phytochem. 2016, 5, 417-420.

16. Heflin, L.E.; Malheiros, R.; Anderson, K.; Johnson, L.K.; Raatz, S.K. Mineral content of eggs differs with hen strain, age, and rearing environment. Poult. Sci. 2018, 97, 1605-1613. [CrossRef] [PubMed]

17. Sokołowicz, Z; Krawczyk, J. Behavior of conservation breed hens kept with or without access to open-air runs. Ann. Anim. Sci. 2007, 1, 153-156.

18. Krawczyk, J. Effect of layer age and egg production level on changes in quality traits of eggs from hens of conservation breeds and commercial hybrids. Ann. Anim. Sci. 2009, 9, 185-193.

19. Krupiński, J. Conservation of livestock genetic resources in Poland. Wiad. Zoot. 2008, 46, I-X. (In Polish)

20. Smulikowska, S.; Rutkowski, A. Poultry Feeding Standards; The Kielanowski Institute of Animal Physiology and Nutrition, Polish Academy of Sciences: Jabłonna, Poland, 2005. (In Polish)

21. AOAC. Official Methods of Analysis, 19th ed.; Association of Official Analytical Chemist: Washington, DC, USA, 2012.

22. Connelly, N.G.; Hartshorn, R.M.; Damhus, T.; Hutton, A.T. Nomenclature of Inorganic Chemistry; IUPAC Recommendations; RSC Publising: Cambridge, UK, 2005.

23. Vernon, R.E. Which Elements Are Metalloids? J. Chem. Educ. 2013, 90, 1703-1707. [CrossRef]

24. Żymankowska-Kumon, S. Criteria for assessing the properties of binding bentonites. Arch. Foundry Engin. 2012, 12, 209-2012. (In Polish) [CrossRef] 
25. Bubel, F.; Dobrzański, Z.; Kowalska-Góralska, M.; Opaliński, S.; Trziszka, T. Effect of mineral-organic feed additives on the content of elements in raw egg material. Przem. Chem. 2013, 92, 962-965.

26. Świątkiewicz, S.; Arczewska-Włosek, A.; Józefiak, D. Application of microalgae biomass in poultry nutrition. World's Poult. Sci. J. 2015, 71, 663-672. [CrossRef]

27. Michalak, I.; Chojnacka, K.; Dobrzanski, Z.; Gorecki, H.; Zielinska, A.; Korczynski, M.; Opalinski, S. Effect of macroalgae enriched with microelements on egg quality parameters and mineral content of eggs, eggshell, blood, feathers and droppings. J. Anim. Physiol. Anim. Nutr. 2012, 95, 374-387. [CrossRef] [PubMed]

28. Gruszecka-Kosowska, A.; Wdowin, M.; Kosowski, T.; Klimek, A. An analysis of the chemistry, mineralogy and texture of waste dolomite powder used to identify its potential application in industry. Geol. Geophys. Environ. 2015, 41, 343-352. [CrossRef]

29. Buckiuniene, V.; Grashorn, M.A.; Gruzauskas, R.; Kliseviciute, V.; Raceviciute-Stupeliene, A.; Svirmickas, G.; Bliznikas, S.; Miezeliene, A.; Alencikiene, G. Effect of organic and inorganic iron in the diet on yolk iron content, fatty acids profile, malondialdehyd concentration, and sensory quality of chicken eggs. Eur. Poult. Sci. 2016, 80. [CrossRef]

30. Dolińska, B.; Opaliński, S.; Zieliński, M.; Chojnacka, K.; Dobrzański, Z.; Ryszka, F. Iodine concentration in fodder influences the dynamics of iodine levels in hen's egg components. Biol. Trace. Elem. Res. 2011, 144, 747-752. [CrossRef] [PubMed]

31. Witkowska, Z.; Świniarska, M.; Korczyński, M.; Opaliński, S.; Konkol, D.; Michalak, I.; Saeid, A.; Mironiuk, M.; Chojnacka, K. Biofortification of hens' eggs with microelements by innovative bio-based dietary supplement. J. Anim. Physiol. Anim. Nutr. 2019, 103, 485-492. [CrossRef] [PubMed]

32. Cywa-Benko, K.; Krawczyk, J.; Wężyk, S. Quality of food eggs obtained from hens of Polish native breeds. Rocz. Nauk. Zoot. 2003, 30, 405-413. (In Polish)

33. Dobrzański, Z.; Trziszka, T.; Herbut, E.; Krawczyk, J.; Tronina, P. Effect of humic preparations on productivity and quality traits of eggs from greenleg partridge hens. Ann. Anim. Sci. 2009, 9, 165-174.

34. Krawczyk, J.; Sokołowicz, Z.; Szymczyk, B. Effect of housing system on cholesterol, vitamin and fatty acid content of yolk and physical characteristics of eggs from Polish native hens. Arch. Geflügelkd. 2011, 75, 151-157.

35. Biesiada-Drzazga, B.; Banaszewska, D.; Andraszek, K.; Bombik, E.; Kałuża, H.; Rojek, A. Comparison of egg quality of free range Araucana and Green-legged Partridge chickens. Arch. Geflügelk. Europ. Poult. Sci. 2014, 78, 1-8.

36. Zhuang, P.; Zou, H.L.; Shu, W.S. Biotransfer of heavy metals along a soil-plant-insect-chicken foodchain: Field study. J. Environ. Sci. 2009, 21, 849-853. [CrossRef]

37. Szablewski, T.; Gornowicz, E.; Stuper-Szablewska, K.; Kaczmarek, A.; Cegielska-Radziejewska, R. Mineral composition of contents in table eggs from autochthonous hen breeds bred under ecological conditions. Żywn-Nauk. Technol. Ja. 2013, 5, 42-51.

38. Holt, P.S.; Davies, R.H.; Dewulf, J.; Gast, R.K.; Huwe, J.K.; Jones, D.R.; Waltman, D.; Willian, K.R. The impact of different housing systems on eggs safety and quality. Poult. Sci. 2011, 90, 251-261. [CrossRef] [PubMed]

39. Nisianakis, P.; Giannenas, I.; Gavriil, A.; Kontopidis, G.; Kyriazakis, I. Variation in trace element contents among chicken, turkey, duck, goose, and pigeon eggs analyzed by inductively coupled plasma mass spectrometry (ICP-MS). Biol. Trace Elem. Res. 2009, 128, 62-71. [CrossRef] [PubMed]

40. Kabeer, M.S.; Hameed, I.; Kashif, S.R.; Khan, M.; Tahir, A.; Anum, F.; Khan, S.; Raza, S. Contamination of heavy metals in poultry eggs: A study presenting relation between heavy metals in feed intake and eggs. Arch. Environ. Occup. Health 2020. [CrossRef]

41. Herman, K.; Korczyński, M.; Janeczek, M.; Wełmiński, P.; Kowalczyk-Zając, M.; Leśków, A.; Całkosiński, I.; Dobrzyńśki, M. Effect of toxic metals on oral tissues. J. Educ. Health Sport 2017, 7, 209-220.

42. Waseem, A.; Arshad, J.; Iqbal, F.; Sajjad, A.; Mehmood, Z.; Murtaza, G. Pollution status of Pakistan: A retrospective review of heavy metal contamination of water, soil and vegetables. BioMed. Res. Int. 2014, 81326. [CrossRef]

43. Khan, A.; Khan, S.; Khan, M.A.; Quamar, Z.; Waqas, M. The uptake and bioaccumulation of heavy metals by food plants, their effects on plants nutrients, and associated health risk: A review. Environ. Sci. Pollut. Res. Int. 2015, 22, 13772-13799. [CrossRef]

44. Quareshi, A.S.; Hussain, M.I.; Ismail, S.; Khan, Q.M. Evaluating heavy metal accumulation and potential health risks in vegetables irrigated with treated wastewater. Chemosphere 2016, 163, 54-61. [CrossRef] [PubMed]

45. Dobrzański, Z.; Korczyński, M.; Chojnacka, K.; Górecki, H.; Opaliński, S. Influence of organic forms of copper, manganese and iron on bioaccumulation of these metals and zinc in laying hens. J. Elementol. 2008, 13, 309-319.

46. García-Barrera, T.; Gómez-Ariza, J.L.; González-Fernández, M.; Moreno, F.; García-Sevillano, M.A.; Gómez-Jacinto, V. Biological responses related to agonistic, antagonistic and synergistic interactions of chemical species. Anal. Bioanal. Chem. 2012, 403, 2237-2253. [CrossRef] [PubMed] 